TRANSACTIONS OF THE

AMERICAN MATHEMATICAL SOCIETY

Volume 360, Number 3, March 2008, Pages 1179-1191

S 0002-9947(07)04202-X

Article electronically published on October 2, 2007

\title{
KLESHCHEV'S DECOMPOSITION NUMBERS AND BRANCHING COEFFICIENTS IN THE FOCK SPACE
}

\author{
JOSEPH CHUANG, HYOHE MIYACHI, AND KAI MENG TAN
}

\begin{abstract}
We give combinatorial descriptions of some coefficients of the canonical basis of the $q$-deformed Fock space representation of $U_{q}(\widehat{\mathfrak{s} l} e)$ and of some matrix entries for the action of the Chevalley generators $f_{r}$ with respect to the canonical basis. These are $q$-analogues of results of Kleshchev on decomposition numbers and branching coefficients for symmetric groups and Schur algebras.
\end{abstract}

\section{INTRODUCTION}

Throughout we fix an integer $e \geq 2$. Lascoux, Leclerc, and Thibon 4, 9] used the representation theory of the quantum affine algebra $U_{q}\left(\widehat{\mathfrak{s l}}_{e}\right)$ to introduce for every pair of partitions $\lambda$ and $\sigma$ a polynomial $d_{\lambda \sigma}(q)$ with integer coefficients (which depends on $e$ ). They conjectured these polynomials to be $q$-analogues of decomposition numbers for Hecke algebras and quantized Schur algebras at complex $e$-th roots of unity. These conjectures were proved by Ariki [1] and by Varagnolo and Vasserot [16] respectively, and these polynomials are now often called $q$-decomposition numbers.

Leclerc's lectures [8] are a good introduction to this subject as well as a convenient reference for the results we need here.

In [6, Theorem 1.10], Kleshchev gave a combinatorial description in terms of what he calls 'latticed subsets' of the decomposition numbers of symmetric groups (i.e., multiplicities of simple modules $D^{\mu}$ in Specht modules $S^{\lambda}$ ) in cases where the partition $\mu$ is obtained from $\lambda$ by moving a single node. He also provided a description in terms of 'normal nodes' of the branching coefficient (i.e., the multiplicity of the simple module $D^{\nu}$ in the restricted simple module $\left.D^{\mu} \downarrow_{\mathfrak{S}_{n-1}}\right)$ when $\nu$ is obtained from $\mu$ by removing a node ([6, Theorem 1.4]). This branching coefficient may also be described as the multiplicity of the projective cover $P\left(D^{\mu}\right)$ in a direct sum decomposition of the induced projective cover $P\left(D^{\nu}\right) \uparrow \mathfrak{S}_{n}$. In this paper, we give analogues of these results for the $q$-decomposition numbers. When we apply Ariki's and Varagnolo-Vasserot's theorems, we then obtain the corresponding decomposition numbers and branching coefficients for Hecke algebras and quantized Schur algebras at complex $e$-th roots of unity.

This paper is organized as follows. In section 2, we introduce the background theory and obtain some useful preliminary results. In section 3, we review the theory of sign sequences and set up the machinery necessary for the proof of the

Received by the editors July 23, 2005.

2000 Mathematics Subject Classification. Primary 17B37; Secondary 20 C08. 
main theorems of this paper. We then state and prove the main theorems in section 4 and finally conclude with an example illustrating the main theorems.

\section{BACKGROUND}

2.1. Partitions. Let $\mathcal{P}_{n}$ be the set of partitions of a nonnegative integer $n$, and let $\mathcal{P}=\bigcup_{n} \mathcal{P}_{n}$ be the set of all partitions. The standard dominance ordering on $\mathcal{P}_{n}$ is denoted by $\unrhd$. We identify a partition $\lambda=\left(\lambda_{1}, \lambda_{2}, \ldots\right)$ with its Young diagram

$$
\left\{(j, k) \in \mathbb{Z}^{+} \times \mathbb{Z}^{+} \mid 1 \leq k \leq \lambda_{j}\right\} .
$$

The residue of a node $(j, k)$ in a Young diagram $\mu$ is the residue class of $(k-j)$ modulo $e$. If $(j, k)$ has residue $r$, we say that $(j, k)$ is an $r$-node. If in removing $(j, k)$ from $\mu$, we obtain a Young diagram $\lambda$, then $(j, k)$ is both a removable $r$-node of $\mu$ and an indent 11 -node of $\lambda$.

2.2. The Fock space representation. The algebra $U_{q}\left(\widehat{\mathfrak{s l}}_{e}\right)$ is the associative algebra over $\mathbb{C}(q)$ with generators $e_{r}, f_{r}, k_{r}, k_{r}^{-1}(0 \leq r \leq e-1), d, d^{-1}$ subject to some relations (see, for example, $[8, \S 4])$. An important $U_{q}\left(\widehat{\mathfrak{s l}}_{e}\right)$-module is the Fock space representation $\mathcal{F}[3$, 14, which has a basis $\{s(\lambda) \mid \lambda \in \mathcal{P}\}$ as a $\mathbb{C}(q)$-vector space. In fact, $\mathcal{F}$ admits another action by the Heisenberg algebra which commutes with the action of $U_{q}\left(\widehat{\mathfrak{s l}}_{e}\right)$, and these two actions in effect make $\mathcal{F}$ a $U_{q}\left(\widehat{\mathfrak{g l}}_{e}\right)$-module.

For our purposes an explicit description of the action of just the $f_{r}$ 's on $\mathcal{F}$ will suffice.

Let $\lambda$ be a partition with indent $r$-node $(j, k)$, and write $\mu$ for the partition obtained from $\lambda$ by adding $(j, k)$. Let $N(\lambda, \mu)$ be the number of indent $r$-nodes of $\lambda$ that are situated to the right of $(j, k)$ minus the number of removable $r$-nodes of $\lambda$ situated to the right of $(j, k)$. We have

$$
f_{r}(s(\lambda))=\sum_{\mu} q^{N(\lambda, \mu)} s(\mu)
$$

where the sum is over all Young diagrams $\mu$ obtained from $\lambda$ by adding an indent $r$-node.

In [9], Leclerc and Thibon introduced an involution $x \mapsto \bar{x}$ on $\mathcal{F}$, having the following properties (among others):

$$
\overline{a(q) x}=a\left(q^{-1}\right) \bar{x} \quad \text { and } \quad \overline{f_{r}(x)}=f_{r}(\bar{x}) \quad(a \in \mathbb{C}(q), x \in \mathcal{F}) .
$$

There is a distinguished basis $\{G(\sigma) \mid \sigma \in \mathcal{P}\}$ of $\mathcal{F}$ having the following characterization ([9, Theorem 4.1]):

(1) $G(\sigma) \equiv s(\sigma)(\bmod q L)$, where $L$ is the $\mathbb{Z}[q]$-lattice in $\mathcal{F}$ generated by $\{s(\lambda) \mid$ $\lambda \in \mathcal{P}\}$

(2) $\overline{G(\sigma)}=G(\sigma)$.

This basis is in fact the canonical basis of $\mathcal{F}$ as a $U_{q}\left(\widehat{\mathfrak{g l}}_{e}\right)$-module in the sense of 9 .

Let $\langle-,-\rangle$ denote the inner product on $\mathcal{F}$ for which $\{s(\lambda) \mid \lambda \in \mathcal{P}\}$ is orthonormal. Then the $q$-decomposition number $d_{\lambda \sigma}(q)$ is defined as $\langle G(\sigma), s(\lambda)\rangle$, the coefficient of $s(\lambda)$ in $G(\sigma)$.

\footnotetext{
${ }^{1}$ We are following the terminlogy used in $[6]$ and $[\underline{8}$; Kleshchev has since used the term addable nodes in more recent papers.
} 
The $q$-decomposition numbers enjoy the following property:

Theorem 2.1 ([15, 3.1], [8, Theorem 9, Proposition 11, Corollary 14]). We have

$$
\begin{aligned}
& d_{\sigma \sigma}(q)=1, \\
& d_{\lambda \sigma}(q) \in q \mathbb{N}_{0}[q] \text { for all } \lambda \neq \sigma .
\end{aligned}
$$

Furthermore, $d_{\lambda \sigma}(q) \neq 0$ only if $\sigma \unrhd \lambda$ and $\lambda$ and $\sigma$ have the same e-core.

The deepest part of this theorem is the positivity of the $q$-decomposition numbers, which follows from Lusztig's geometric approach to canonical bases. We shall require another positivity property of the canonical basis of $\mathcal{F}$. While it is a direct consequence of Lusztig's work [11, 12] and Schiffmann's solution [15] to VaragnoloVasserot's conjecture [16], we could not find a convenient reference, so we briefly review the argument here.

Let $\mathbf{U}_{e}^{-}$be the generic Hall algebra of type $A_{e-1}^{(1)}$. It contains the negative part of $U_{q}\left(\widehat{\mathfrak{s l}}_{e}\right)$ as a proper subalgebra. Lusztig [11] defines a canonical basis $\mathbf{B}$ of $\mathbf{U}_{e}^{-}$ in terms of perverse sheaves.

Theorem 2.2 (Lusztig). For any two canonical basis elements $\mathbf{b}$ and $\mathbf{b}^{\prime}$ of $\mathbf{U}_{e}^{-}$, the coefficients of the expansion of $\mathbf{b b}^{\prime}$ in terms of the canonical basis belong to $\mathbb{N}_{0}\left[q, q^{-1}\right]$.

This result is stated explicitly by Lusztig [11, 11.5(a)], at least for the canonical basis in the negative part of $U_{q}\left(\widehat{\mathfrak{s l}}_{e}\right)$; the argument for the Hall algebra is the same. To explain this we use the notation in the proof of [16, 7.5]. The point is that the convolution $*$ is defined by Lusztig on the categories $\mathcal{D}_{G_{U}}^{s s}\left(E_{U}\right)$ themselves. So the convolution product of simple perverse sheaves on $E_{U}$ and $E_{W}$ is a direct sum of shifts of simple perverse sheaves on $E_{V}$. As the elements of $\mathbf{B}$ are defined (see, e.g., $[16,3.5])$ as Frobenius traces of simple perverse sheaves on various $E_{U}$ 's, we get the claim.

The Chevalley generators $f_{r}$ of the negative part of $U_{q}\left(\widehat{\mathfrak{s l}}_{e}\right)$ are, via the embedding into $\mathbf{U}_{e}^{-}$, elements of $\mathbf{B}$ (see, e.g., [16, 7.5]). Hence we have

Corollary 2.3. For any canonical basis element $\mathbf{b} \in \mathbf{B}$ and any Chevalley generator $f_{r}$ of $\mathbf{U}_{e}^{-}$, each coefficient of the expansion of $f_{r} \mathbf{b}$ in terms of the canonical basis $\mathbf{B}$ belongs to $\mathbb{N}_{0}\left[q, q^{-1}\right]$.

Now Varagnolo and Vasserot [16, 6.2] described an action of the Hall algebra $\mathbf{U}_{e}^{-}$on the Fock space $\mathcal{F}$ extending the action of the negative part of $U_{q}\left(\widehat{\mathfrak{s l}}_{e}\right)$, and their conjecture that

$$
\mathbf{B} s(\emptyset)=\{G(\lambda) \mid \lambda \in \mathcal{P}\}
$$

was proved by Schiffmann 15. We deduce the following positivity result.

Proposition 2.4. If we write $f_{r}(G(\sigma))=\sum_{\rho} a_{\rho}(q) G(\rho)$, then $a_{\rho}(q) \in \mathbb{N}_{0}\left[q, q^{-1}\right]$ for all $\rho$. We also have $a_{\rho}(q)=a_{\rho}\left(q^{-1}\right)$.

Proof. For $\sigma \in \mathcal{P}$ choose $\mathbf{b}_{\sigma} \in \mathbf{B}$ such that $\mathbf{b}_{\sigma} s(\emptyset)=G(\sigma)$. Then, by Corollary 2.3 we can write

$$
f_{r} \mathbf{b}_{\sigma}=\sum_{m} c_{m}(q) \mathbf{b}_{m}
$$


where $c_{m}(q) \in \mathbb{N}_{0}\left[q, q^{-1}\right]$. Since

$$
\sum_{m} c_{m}(q) \mathbf{b}_{m} s(\emptyset)=f_{r} \mathbf{b}_{\sigma} s(\emptyset)=f_{r} G(\sigma)
$$

and each $\mathbf{b}_{m} s(\emptyset)=G(\rho)$ for some $\rho$, we are done with the first assertion. The second assertion follows from the fact that $f_{r}(G(\sigma))$ and $G(\rho)$ are all bar-invariant.

2.3. Some other useful results. We collate together some results which we shall require.

Theorem 2.5 ([2, Theorem 1]). Let $\lambda=\left(\lambda_{1}, \lambda_{2}, \ldots\right)$ and $\mu=\left(\mu_{1}, \mu_{2}, \ldots\right)$ be partitions.

(1) (Row removal) Suppose that $\lambda_{1}+\cdots+\lambda_{r}=\mu_{1}+\cdots+\mu_{r}$ for some $r$ and let

$$
\begin{array}{llrl}
\lambda^{(0)} & =\left(\lambda_{1}, \ldots, \lambda_{r}\right), & & \mu^{(0)}=\left(\mu_{1}, \ldots, \mu_{r}\right), \\
\lambda^{(1)}=\left(\lambda_{r+1}, \lambda_{r+2}, \ldots\right), & \mu^{(1)}=\left(\mu_{r+1}, \mu_{r+2}, \ldots\right) .
\end{array}
$$

Then $d_{\lambda \mu}(q)=d_{\lambda^{(0)} \mu^{(0)}}(q) d_{\lambda^{(1)} \mu^{(1)}}(q)$.

(2) (Column removal) Suppose that $\lambda_{1}^{\prime}+\cdots+\lambda_{r}^{\prime}=\mu_{1}^{\prime}+\cdots+\mu_{r}^{\prime}$ for some $r$ and let

$$
\begin{array}{ll}
\lambda^{(0) \prime}=\left(\lambda_{1}^{\prime}, \ldots, \lambda_{r}^{\prime}\right), & \mu^{(0) \prime}=\left(\mu_{1}^{\prime}, \ldots, \mu_{r}^{\prime}\right), \\
\lambda^{(1)^{\prime}}=\left(\lambda_{r+1}^{\prime}, \lambda_{r+2}^{\prime}, \ldots\right), & \mu^{(1) \prime}=\left(\mu_{r+1}^{\prime}, \mu_{r+2}^{\prime}, \ldots\right) .
\end{array}
$$

Then $d_{\lambda \mu}(q)=d_{\lambda^{(0)} \mu^{(0)}}(q) d_{\lambda^{(1)} \mu^{(1)}}(q)$.

The following lemma is clear.

Lemma 2.6. Suppose $a_{1}(q)+f_{1}(q)=a_{2}(q)+f_{2}(q)$, with $a_{1}, a_{2} \in \mathbb{C}\left[q, q^{-1}\right]$, $a_{1}\left(q^{-1}\right)=a_{1}(q), a_{2}\left(q^{-1}\right)=a_{2}(q)$ and $f_{1}, f_{2} \in q \mathbb{C}[q]$. Then $a_{1}=a_{2}$ and $f_{1}=f_{2}$.

Proposition 2.7. Suppose $f_{r}(G(\lambda))=\sum_{\nu} a_{\nu}(q) G(\nu)$ and $\left\langle a_{\mu}(q) G(\mu), s(\widehat{\lambda})\right\rangle \neq 0$ where $\widehat{\lambda}$ is the partition obtained from $\lambda$ by adding an indent $r$-node lying on row $b$. Then $\mu$ is obtained from $\lambda$ by adding $k+1$ indent $r$-nodes, on rows $a_{0}, a_{1}, a_{2}, \ldots, a_{k}$ say, and removing $k$ removable $r$-nodes, on rows $b_{1}, b_{2}, \ldots, b_{k}$ say, $(k \geq 0)$ with

$$
a_{0}<b_{1}<a_{1}<b_{2}<a_{2}<\cdots<b_{k}<a_{k} \leq b .
$$

Proof. As $\left\langle a_{\mu}(q) G(\mu), s(\widehat{\lambda})\right\rangle \neq 0$, we have $a_{\mu}(q), d_{\widehat{\lambda} \mu}(q) \neq 0$, so that $\mu \unrhd \widehat{\lambda}$ by Theorem 2.1, Furthermore, $\left\langle f_{r}(G(\lambda)), s(\mu)\right\rangle \neq 0$ by Proposition 2.4 Since

$$
\left\langle f_{r}(G(\lambda)), s(\mu)\right\rangle=\sum_{\widetilde{\mu}} d_{\widetilde{\mu} \lambda}(q)\left\langle f_{r}(s(\widetilde{\mu})), s(\mu)\right\rangle,
$$

where the sum runs over all partitions $\widetilde{\mu}$ obtained from $\mu$ by removing a removable $r$-node, we have $d_{\widetilde{\mu} \lambda}(q) \neq 0$ for at least one such $\widetilde{\mu}$. Fix one such $\widetilde{\mu}$, say the one obtained from $\mu$ by removing the $r$-node on row $a$. We have $\lambda \unrhd \widetilde{\mu}$ by Theorem 2.1, and together with $\mu \unrhd \widehat{\lambda}$, we see that

$$
\sum_{i=1}^{j} \lambda_{i} \geq \sum_{i=1}^{j} \widetilde{\mu}_{i} \geq \sum_{i=1}^{j} \lambda_{i}
$$


whenever $j<\min (a, b)$ or $j \geq \max (a, b)$, so that $\lambda_{i}=\widetilde{\mu}_{i}$ whenever $i<\min (a, b)$ or $i>\max (a, b)$. Furthermore, if $b<a$, then

$$
\sum_{i=1}^{b} \lambda_{i} \geq \sum_{i=1}^{b} \widetilde{\mu}_{i} \geq \sum_{i=1}^{b} \lambda_{i}+1
$$

giving $\lambda_{b} \geq \widetilde{\mu}_{b} \geq \lambda_{b}+1$, a contradiction. Thus $b \geq a$. Furthermore, for each $a \leq j<b$, we have $\sum_{i=a}^{j} \widetilde{\mu}_{i}+1 \geq \sum_{i=a}^{j} \lambda_{i} \geq \sum_{i=a}^{j} \widetilde{\mu}_{i}$, which simplifies to

$$
0 \leq \sum_{i=a}^{j}\left(\lambda_{i}-\widetilde{\mu}_{i}\right) \leq 1
$$

Hence, we conclude that $\widetilde{\mu}$ is obtained from $\lambda$ by adding $k$ indent nodes, on rows $a_{1}, a_{2}, \ldots, a_{k}$ say, and removing $k$ removable nodes, on rows $b_{1}, b_{2}, \ldots, b_{k}$ say, with

$$
a \leq b_{1}<a_{1}<b_{2}<a_{2}<\cdots<b_{k}<a_{k} \leq b .
$$

It remains to show that all the removable and indent nodes involved have residue $r$.

Let $\lambda^{(i)}=\left(\lambda_{b_{i}}, \lambda_{b_{i}+1}, \ldots, \lambda_{a_{i}}\right)$ and $\widetilde{\mu}^{(i)}=\left(\widetilde{\mu}_{b_{i}}, \widetilde{\mu}_{b_{i}+1}, \ldots, \widetilde{\mu}_{a_{i}}\right)$ for each $1 \leq i \leq$ $k$. Then since $d_{\widetilde{\mu} \lambda}(q) \neq 0$, we have $d_{\widetilde{\mu}^{(i)} \lambda^{(i)}}(q) \neq 0$ for all $i$ by the row removal theorem (Theorem 2.5(1)). Thus, for each $i, \widetilde{\mu}^{(i)}$ and $\lambda^{(i)}$ have the same $e$-core by Theorem 2.1, and hence the nodes $\left(b_{i}, \lambda_{b_{i}}\right)$ and $\left(a_{i}, \lambda_{a_{i}}+1\right)$ have the same residue. As $\hat{\lambda}$ is obtained from $\mu$ by adding indent nodes on rows $b_{1}, b_{2}, \ldots, b_{k}, b$ and removing removable nodes on rows $a, a_{1}, a_{2}, \ldots, a_{k}$, and $d_{\widehat{\lambda} \mu}(q) \neq 0$, we can apply an argument similar to the above to conclude that the nodes $\left(b_{i}, \mu_{b_{i}}+1\right)$ and $\left(a_{i-1}, \mu_{a_{i-1}}\right)$ have the same residue, where $b_{k+1}=b$ and $a_{0}=a$. But

$$
\begin{aligned}
\left(b_{i}, \mu_{b_{i}}+1\right) & = \begin{cases}\left(b_{i}, \lambda_{b_{i}}\right), & \text { if } i \leq k, \\
\left(b, \lambda_{b}+1\right), & \text { if } i=k+1\end{cases} \\
\left(a_{i-1}, \mu_{a_{i-1}}\right) & = \begin{cases}\left(a_{i-1}, \lambda_{a_{i-1}+1}\right), & \text { if } i \geq 2, \\
\left(a, \mu_{a}\right), & \text { if } i=1\end{cases}
\end{aligned}
$$

Thus all the nodes involved have the same residue, which must be $r$ as this is the residue of $\left(b, \lambda_{b}+1\right)$ and $\left(a, \mu_{a}\right)$.

\section{Sign SEQUenCES}

In this section, we review the theory of sign sequences introduced by Kleshchev in [6] and set up the machinery necessary for the proof of the main theorems of the next section.

Definition 3.1. Let $T=\left(t_{1}, \ldots, t_{u}\right)$ be a finite sequence with each $t_{v} \in\{ \pm 1\}$. We call $T$ a sign sequence.

For $0 \leq i<j \leq u+1$, we denote the sign subsequence $\left(t_{i+1}, t_{i+2}, \ldots, t_{j-1}\right)$ of $T$ by $T_{i}^{j}$. (Thus, $T_{0}^{u+1}=T$.)

The set (of integers) associated to $T_{i}^{j}$, denoted by $S\left(T_{i}^{j}\right)$, is $\{i+1, i+2, \ldots, j-1\}$.

We also write $|T|$ for $\sum_{i=1}^{u} t_{i}$.

Note. Our notation $T_{i}^{j}$ equals the notation $T(i+1, j-1)$ used by Kleshchev in [6].

We pair up the elements of $S(T)$ using the following algorithm:

(1) $v$ is paired with $v+1$ whenever $t_{v}=1$ and $t_{v+1}=-1$; 
(2) whenever $v$ and $w$ are as yet unpaired, with $v<w, t_{v}=1$ and $t_{w}=-1$, and $v+1, v+2, \ldots, w-1$ are all paired, we pair $v$ with $w$.

Definition 3.2. Denote the sets of paired and unpaired $v \in S(T)$ by $P(T)$ and $U(T)$ respectively. Furthermore, for $\Delta \in\{S, P, U\}$, write $\Delta^{+}(T)$ for $\{v \in \Delta(T) \mid$ $\left.t_{v}=1\right\}$, and similarly define $\Delta^{-}(T)$.

The pairing of elements of $S(T)$ induces an involution $p_{T}$ on $P(T)$.

Example. Let $T=(1,1,-1,-1,-1,1,-1,1)$. Then $U^{-}(T)=\{5\}, U^{+}(T)=\{8\}$, $P^{-}(T)=\{3,4,7\}, P^{+}(T)=\{1,2,6\}$, and $p_{T}(1)=4, p_{T}(2)=3, p_{T}(6)=7$.

We note the following easy consequences arising from this pairing:

\section{Lemma 3.3.}

(1) If $v \in P^{+}(T)$ and $v<w<p_{T}(v)$, then $w \in P(T)$ with $v<p_{T}(w)<p_{T}(v)$.

(2) If $v, w \in P^{+}(T)$ with $v<w$, then either $v<w<p_{T}(w)<p_{T}(v)$ or $v<p_{T}(v)<w<p_{T}(w)$.

(3) For all $v \in U^{-}(T)$ and for all $w \in U^{+}(T)$, we have $v<w$.

(4) $|T|=\left|U^{+}(T)\right|-\left|U^{-}(T)\right|$.

Given a subsequence $T_{i}^{j}$ of $T$, we can also pair the elements of $S\left(T_{i}^{j}\right)$ using the same algorithm. We note that this process is just a 'restriction' of the pairing of elements of $S(T)$. More precisely, we have

$$
P\left(T_{i}^{j}\right)=\left\{v \in P(T) \mid i<v, p_{T}(v)<j\right\} .
$$

Definition 3.4. We say that $T_{i}^{j}$ is latticed if $U^{-}\left(T_{i}^{j}\right)=\emptyset$ (equivalently, if $S^{-}\left(T_{i}^{j}\right)=$ $\left.P^{-}\left(T_{i}^{j}\right)\right)$.

Note. The empty sign sequence is latticed.

Lemma 3.5. Let $T=\left(t_{1}, t_{2}, \ldots, t_{u}\right)$ be a sign sequence, and suppose $v \in S^{+}(T)$. Then $v \in U^{+}(T)$ if and only if $T_{v}^{u+1}$ is latticed.

Proof. If $T_{v}^{u+1}$ is latticed, then $v$ has no pair in $S(T)$, i.e., $v \in U^{+}(T)$. Conversely, if $v \in U^{+}(T)$, and $w \in S^{-}\left(T_{v}^{u+1}\right)$, then $w \in P^{-}(T)$ by Lemma 3.3(3). Furthermore, by Lemma $3.3(1), v<p_{T}(w)$, so that $w \in P^{-}\left(T_{v}^{u+1}\right)$.

Lemma 3.6. If $T_{i}^{j}$ is latticed, then $T_{i}^{v}$ is latticed for all $v$ with $i<v \leq j$.

Proof. If $w \in S^{-}\left(T_{i}^{v}\right)$, then $w \in S^{-}\left(T_{i}^{j}\right)=P^{-}\left(T_{i}^{j}\right)$. Thus $i<p_{T}(w)<w<v$, so that $w \in P^{-}\left(T_{i}^{v}\right)$.

Definition 3.7. A set $A=\left\{a_{1}, a_{2}, \ldots, a_{s}\right\}\left(a_{1}<a_{2}<\cdots<a_{s}\right)$ is a latticed subset for $T_{i}^{j}$ if the following conditions hold:

(1) $A \subseteq S^{-}\left(T_{i}^{j}\right)$;

(2) $T_{a_{k}}^{a_{k+1}}$ is latticed for all $0 \leq k \leq s$ (where $a_{0}=i$ and $a_{s+1}=j$ ).

We allow the possibility of $s=0$ which corresponds to the case $A=\emptyset$.

Note. If $w \in U^{-}\left(T_{i}^{j}\right)$, then $w \in U^{-}\left(T_{k}^{l}\right)$ for all $i \leq k<w<l \leq j$. Thus any latticed subset for $T_{i}^{j}$ necessarily contains $U^{-}\left(T_{i}^{j}\right)$ as a subset.

Remark. Our definitions of latticed sign sequences and latticed subsets are equivalent to those given in Definitions 1.2 and 1.8 of [6] respectively. 
As an immediate consequence of Lemma 3.6, we have

Corollary 3.8. If $A$ is a latticed subset for $T_{i}^{j}$, then $A \cap S\left(T_{i}^{v}\right)$ is a latticed subset for $T_{i}^{v}$ for all $v$ with $i<v \leq j$.

Proposition 3.9. Let $A=\left\{a_{1}, a_{2}, \ldots, a_{s}\right\} \subseteq S^{-}\left(T_{i}^{j}\right)\left(a_{1}<a_{2}<\cdots<a_{s}\right)$. Then

$$
\bar{A}=A \cup \bigcup_{k=0}^{s} U^{-}\left(T_{a_{k}}^{a_{k+1}}\right)
$$

is a latticed subset for $T_{i}^{j}$ (where $a_{0}=i$ and $a_{s+1}=j$ ), and is the unique minimal latticed subset for $T_{i}^{j}$ containing $A$.

We call $\bar{A}$ the closure of $A$ for $T_{i}^{j}$.

Proof. It is easy to see that $\bar{A}$ is a latticed subset for $T_{i}^{j}$. Let $B$ be a latticed subset for $T_{i}^{j}$ containing $A$. If $w \in U^{-}\left(T_{a_{k}}^{a_{k+1}}\right)$, then $w \in U^{-}\left(T_{l_{1}}^{l_{2}}\right)$ for all $a_{k} \leq l_{1}<w<$ $l_{2} \leq a_{k+1}$; thus $w \in B$. This shows that $\bar{A} \subseteq B$.

Definition 3.10. Let $N^{-}(T)$ denote the set of maximal elements of latticed subsets for $T$, i.e.,

$$
N^{-}(T)=\left\{v \in S^{-}(T) \mid v=\max (A) \text { for some latticed subset } A \text { for } T\right\} .
$$

\section{Lemma 3.11.}

(1) We have $v \in N^{-}(T)$ if and only if $v \in S^{-}(T)$ and $T_{v}^{u+1}$ is latticed.

(2) If $U^{-}(T) \neq \emptyset$, then $\max \left(U^{-}(T)\right) \in N^{-}(T)$. Moreover, $w \notin N^{-}(T)$ for all other $w \in U^{-}(T)$.

(3) If $x \in S(T)$, then $N^{-}(T) \cap S\left(T_{x}^{u+1}\right)=N^{-}\left(T_{x}^{u+1}\right)$.

Proof. For part (1), the forward direction is clear from the definition; conversely, if $T_{v}^{u+1}$ is latticed, then $v$ is the maximal element of the closure of $\{v\}$ in $T$. Part (2) follows since $U^{-}(T)$ is the closure of $\emptyset$ for $T$, while part (3) follows immediately from part (1).

Given a latticed subset $A$ for $T_{0}^{w}$ with $w \in S^{+}(T)$, write $\widehat{A}$ for $A \cup U^{-}\left(T_{w}^{u+1}\right)$. Then $\widehat{A}$ is a latticed subset for $T$. Note that, by Lemma 3.5, we have $\widehat{A}=\emptyset$ if and only if $A=\emptyset$ and $w \in U^{+}(T)$.

Given a nonempty latticed subset $B$ for $T$, write $\tau(B)$ for $B \backslash\{\max (B)\}$. Then $\tau(B)$ is a latticed subset for $T_{0}^{\max (B)}$ by Corollary 3.8, Let

$$
\begin{aligned}
L^{+}=\left\{(A, w) \mid A \text { is a latticed subset for } T_{0}^{w}, w \in S^{+}(T)\right\} \\
\\
\backslash\left\{(\emptyset, w) \mid w \in U^{+}(T)\right\}, \\
L^{-}=\left\{(B, x) \mid B \text { is a latticed subset for } T_{0}^{x}, x \in N^{-}(T)\right\} .
\end{aligned}
$$

Note that if $(B, x) \in L^{-}$, then $B \cup\{x\}$ is a latticed subset for $T$; in particular, $U^{-}(T) \subseteq B \cup\{x\}$, with equality if and only if

$$
(B, x)=\left(\tau\left(U^{-}(T)\right), \max \left(U^{-}(T)\right)\right) .
$$

Proposition 3.12. Let $\alpha: L^{+} \rightarrow L^{-},(A, w) \mapsto(\tau(\widehat{A}), \max (\widehat{A}))$ and let $(B, x) \in$ $L^{-}$. Then

$$
\alpha^{-1}\{(B, x)\}=\left\{(B \cup\{x\}, w) \mid w \in U^{+}(T), w>x\right\} \cup M,
$$

where $M=\left\{\left(B \cap S\left(T_{0}^{w_{0}}\right), w_{0}\right)\right\}$ with $w_{0}=\max \left\{y \in P^{+}(T) \mid p_{T}(y) \in B \cup\{x\}\right\}$ unless $B \cup\{x\}=U^{-}(T)$, in which case $M=\emptyset$. 
Proof. Observe first that $\alpha(A, w)=(B, x)$ if and only if

$$
\widehat{A}=A \cup U^{-}\left(T_{w}^{u+1}\right)=B \cup\{x\} .
$$

If $w \in U^{+}(T)$, then this condition reduces to $A=B \cup\{x\}$, since $U^{-}\left(T_{w}^{u+1}\right)=\emptyset$ by Lemma 3.5 thus, $\alpha(A, w)=(B, x)$ if and only if $A=B \cup\{x\}$ and $x<w$. Furthermore, if $\alpha\left(A_{1}, w_{1}\right)=(B, x)=\alpha\left(A_{2}, w_{2}\right)$ for some $w_{1}, w_{2} \in P^{+}(T)$ with $w_{1}<w_{2}$, then $p_{T}\left(w_{2}\right)>w_{2}>w_{1}$, so that $p_{T}\left(w_{2}\right) \in \widehat{A_{2}} \backslash \widehat{A_{1}}$, contradicting $\widehat{A_{1}}=B \cup\{x\}=\widehat{A_{2}}$. Thus, there exists at most one $(A, w)$ with $w \in P^{+}(T)$ such that $\alpha(A, w)=(B, x)$. As $p_{T}(w) \in \widehat{A} \backslash U^{-}(T)$ if $w \in P^{+}(T)$, we see that there does not exist $(A, w)$ with $w \in P^{+}(T)$ such that $\alpha(A, w)=(B, x)$ if $B \cup\{x\}=U^{-}(T)$. It remains then to show that $\alpha\left(A_{0}, w_{0}\right)=(B, x)$ whenever $B \cup\{x\} \neq U^{-}(T)$, where $A_{0}=B \cap S\left(T_{0}^{w_{0}}\right)$.

By Corollary 3.8, $A_{0}$ is a latticed subset of $T_{0}^{w_{0}}$. Since $U\left(T_{w_{0}}^{p_{T}\left(w_{0}\right)}\right)=\emptyset$ by Lemma 3.3 (1), we have

$$
U^{-}\left(T_{w_{0}}^{u+1}\right)=\left\{p_{T}\left(w_{0}\right)\right\} \cup U^{-}\left(T_{p_{T}\left(w_{0}\right)}^{u+1}\right) .
$$

Thus $\widehat{A_{0}}=A_{0} \cup U^{-}\left(T_{w_{0}}^{u+1}\right)$ is the closure of $A_{0} \cup\left\{p_{T}\left(w_{0}\right)\right\}$ for $T$, and since $A_{0} \cup$ $\left\{p_{T}\left(w_{0}\right)\right\} \subseteq B \cup\{x\}$, we have $\widehat{A_{0}} \subseteq B \cup\{x\}$ by Proposition 3.9. Conversely, if $b \in B \cup\{x\} \backslash A_{0}$, then $b>w_{0}$. If $b \in P^{-}(T)$, then $p_{T}(b) \leq w_{0}$ by maximality of $w_{0}$, so that $b \in U^{-}\left(T_{w_{0}}^{u+1}\right)$. On the other hand, if $b \in U^{-}(T)$, then certainly $b \in U^{-}\left(T_{w_{0}}^{u+1}\right)$. Thus $B \cup\{x\} \backslash A_{0} \subseteq U^{-}\left(T_{w_{0}}^{u+1}\right)$, so that $B \cup\{x\} \subseteq A_{0} \cup U^{-}\left(T_{w_{0}}^{u+1}\right)=\widehat{A_{0}}$. Hence $\widehat{A_{0}}=B \cup\{x\}$.

In the corollary below, we use the notation $[k]$ to denote $q^{1-k}+q^{3-k}+\cdots+$ $q^{k-3}+q^{k-1}$ for $k \in \mathbb{Z}^{+}$.

Corollary 3.13. Let $(B, x) \in L^{-}$. Then

$$
\sum_{(A, w) \in \alpha^{-1}\{(B, x)\}} q^{1+2|A|+\left|T_{0}^{w}\right|-\left|T_{w}^{u+1}\right|}=\left[1+\left|T_{x}^{u+1}\right|\right] q^{1+2|B|+\left|T_{0}^{x}\right|}-\varepsilon,
$$

where $\varepsilon=0$ unless $B \cup\{x\}=U^{-}(T)$, in which case $\varepsilon=q^{-|T|}$.

Proof. By Proposition 3.12, for each $(A, w) \in \alpha^{-1}\{(B, x)\}$ with $w \in U^{+}(T)$, we have $w>x$ and $A=B \cup\{x\}$, so that

$$
q^{1+2|A|+\left|T_{0}^{w}\right|-\left|T_{w}^{u+1}\right|}=q^{1+2|B|+\left|T_{0}^{x}\right|+\left|T_{x}^{u+1}\right|-2\left|T_{w}^{u+1}\right|} .
$$

Moreover, the only other possible element of $\alpha^{-1}\{(B, x)\}$ is $\left(B \cap S\left(T_{0}^{w_{0}}\right), w_{0}\right)$ where $w_{0}=\max \left\{y \in P^{+}(T) \mid p_{T}(y) \in B \cup\{x\}\right\}$, and this contributes

$$
q^{1+2|B|+\left|T_{0}^{x}\right|-\left|T_{x}^{u+1}\right|}
$$

to the sum. Note further that if $B \cup\{x\}=U^{-}(T)$, then

$$
q^{1+2|B|+\left|T_{0}^{x}\right|-\left|T_{x}^{u+1}\right|}=q^{-|T|} .
$$

The corollary thus follows. 


\section{MAin Results}

Throughout this section, we fix a residue class $r$ modulo $e$. We denote the set of indent (resp. removable) $r$-nodes of a partition $\lambda$ by $I(\lambda)$ (resp. $R(\lambda)$ ). We label the elements of $I(\lambda) \cup R(\lambda)$ as follows: $I(\lambda) \cup R(\lambda)=\left\{c_{1}, c_{2}, \ldots, c_{u}\right\}$ such that if $c_{v}$ is situated to the left of $c_{w}$ then $v<w$. The partition $\lambda$ induces a sign sequence $T(\lambda)=\left(t_{1}, t_{2}, \ldots, t_{u}\right)$ where $t_{v}=1$ if $c_{v} \in R(\lambda)$, and $t_{v}=-1$ if $c_{v} \in I(\lambda)$. If $c_{v} \in I(\lambda)$ (resp. $R(\lambda)$ ), we denote the partition obtained from $\lambda$ by adding (resp. removing) $c_{v}$ as $\lambda \uparrow^{v}$ (resp. $\lambda \downarrow_{v}$ ).

In the statements of the main theorems below, $\lambda \in \mathcal{P}$ with $T(\lambda)=\left(t_{1}, \ldots, t_{u}\right)$, and $[k]=q^{1-k}+q^{3-k}+\cdots+q^{k-3}+q^{k-1}$ for any $k \in \mathbb{Z}^{+}$.

Theorem 4.1. Suppose $f_{r}(G(\lambda))=\sum_{\mu} a_{\mu}(q) G(\mu)$. Let $v \in S^{-}(T(\lambda))$. Then

$$
a_{\lambda \uparrow^{v}}(q)= \begin{cases}{\left[1+\left|T(\lambda)_{v}^{u+1}\right|\right],} & \text { if } v \in N^{-}(T(\lambda)) ; \\ 0, & \text { otherwise. }\end{cases}
$$

Furthermore, if $i_{k} \in S^{-}(T(\lambda)), j_{k} \in S^{+}(T(\lambda))$ for $k=1,2, \ldots, s$ with $i_{1}<j_{1}<$ $i_{2}<j_{2}<\cdots<i_{s}<j_{s}<v$, then

$$
a_{\lambda \uparrow^{i_{1}} \downarrow_{j_{1}} \ldots \uparrow^{i_{s}} \downarrow_{j_{s}} \uparrow^{v}}(q)=0 .
$$

Remark. Note that $a_{\mu}(q)$ is the $q$-analogue of branching coefficients. The first assertion of Theorem 4.1 agrees with Theorem 1.4 of [6] upon specialization at $q=1$ with $e=p$ and $\lambda p$-regular, since a removable $r$-node $c_{w}$ of $\lambda$ is normal if and only if $T(\lambda)_{w}^{u+1}$ is latticed, so that $c_{v}$ is a normal node of $\lambda \uparrow^{v}$ if and only if $v \in N^{-}(T(\lambda))$ by Lemma 3.11(1).

Theorem 4.2. Let $v \in S^{-}(T(\lambda))$ and $w \in S^{+}(T(\lambda))$ with $v<w$. Then

$$
d_{\lambda \uparrow^{v} \downarrow_{w}, \lambda}(q)=\sum_{A} q^{1+2|A|+\left|T(\lambda)_{v}^{w}\right|}
$$

where the sum runs over all latticed subsets $A$ for $T(\lambda)_{v}^{w}$.

Proof. We prove Theorems 4.1 and 4.2 simultaneously by induction. For $\lambda=\emptyset \in$ $\mathcal{P}_{0}$, Theorem 4.2 holds trivially.

Let $\lambda \in \mathcal{P}_{n}$ and $v \in S^{-}(T(\lambda))$, and suppose that Theorem 4.2 holds for all partitions $\mu \in \mathcal{P}_{m}$ with $m \leq n$, and Theorem 4.1 holds for all $x \in S^{-}(T(\lambda))$ with $x>v$. Let $f_{r}(G(\lambda))=\sum_{\mu} \bar{a}_{\mu}(q) G(\mu)$. Write $T$ for $T(\lambda)_{v}^{u+1}$.

By Proposition 2.7, Lemma 3.11(3) and the induction hypothesis, we have

$$
\begin{aligned}
\left\langle f_{r}(G(\lambda)), s\left(\lambda \uparrow^{v}\right)\right\rangle & =\sum_{\mu}\left\langle a_{\mu}(q) G(\mu), s\left(\lambda \uparrow^{v}\right)\right\rangle \\
& =\sum_{x \in N^{-}(T) \cup\{v\}}\left\langle a_{\lambda \uparrow^{x}}(q) G\left(\lambda \uparrow^{x}\right), s\left(\lambda \uparrow^{v}\right)\right\rangle \\
& =a_{\lambda \uparrow^{v}}(q)+\sum_{x \in N^{-}(T)}\left[1+\left|T(\lambda)_{x}^{u+1}\right|\right] d_{\lambda \uparrow^{v}, \lambda \uparrow^{x}}(q) .
\end{aligned}
$$


On the other hand, we also have

$$
\begin{aligned}
\left\langle f_{r}(G(\lambda)), s\left(\lambda \uparrow^{v}\right)\right\rangle & =\left\langle f_{r}(s(\lambda)), s\left(\lambda \uparrow^{v}\right)\right\rangle+\sum_{w \in S^{+}(T)} d_{\lambda \uparrow^{v} \downarrow_{w}, \lambda}(q)\left\langle f_{r}\left(s\left(\lambda \uparrow^{v} \downarrow_{w}\right)\right), s\left(\lambda \uparrow^{v}\right)\right\rangle \\
& =q^{-|T|}+\sum_{w \in S^{+}(T)} \sum_{A} q^{1+2|A|+\left|T(\lambda)_{v}^{w}\right|-\left|T(\lambda)_{w}^{u+1}\right|},
\end{aligned}
$$

where $A$ runs over all the latticed subsets for $T(\lambda)_{v}^{w}$.

If $v \notin N^{-}(T(\lambda))$, then $U^{-}(T) \neq \emptyset$ by Lemma $3.11(1)$, so that $\emptyset$ is not a latticed subset for $T_{v}^{w}$ for all $w \in U^{+}(T)$. By Corollary 3.13 (1) can be rewritten as

$$
\sum_{x \in N^{-}(T)} \sum_{B}\left[1+\left|T(\lambda)_{x}^{u+1}\right|\right] q^{1+2|B|+\left|T(\lambda)_{v}^{x}\right|},
$$

where $B$ runs over all the latticed subsets for $T(\lambda)_{v}^{x}$. By the induction hypothesis and the row removal theorem (Theorem 2.5(1)), we have

$$
\sum_{B} q^{1+2|B|+\left|T(\lambda)_{v}^{x}\right|}=d_{\lambda \uparrow^{v}, \lambda \uparrow^{x}}(q),
$$

except possibly when $x$ indexes the indent $r$-node in the top row of $\lambda$. Comparing (2) with $(*)$ and using Lemma 2.6. we see that $a_{\lambda \uparrow^{v}}(q)=0$, and $\sum_{B} q^{1+2|B|+\left|T(\lambda)_{v}^{x}\right|}=$ $d_{\lambda \uparrow^{v}, \lambda \uparrow^{x}}(q)$ even when $x$ indexes the indent $r$-node in the top row of $\lambda$.

If $v \in N^{-}(T(\lambda))$, then $U^{-}(T)=\emptyset$ by Lemma 3.11(1), and hence $\emptyset$ is a latticed subset for $T(\lambda)_{v}^{w}$ for all $w \in U^{+}(T)$. Thus, by Corollary 3.13, (1) can be rewritten as

$$
\begin{array}{r}
q^{-|T|}+\sum_{w \in U^{+}(T)} q^{1+\left|T(\lambda)_{v}^{w}\right|-\left|T(\lambda)_{w}^{u+1}\right|}+\sum_{x \in N^{-}(T)} \sum_{B}\left[1+\left|T(\lambda)_{x}^{u+1}\right|\right] q^{1+2|B|+\left|T(\lambda)_{v}^{x}\right|} \\
=[1+|T|]+\sum_{x \in N^{-}(T)} \sum_{B}\left[1+\left|T(\lambda)_{x}^{u+1}\right|\right] q^{1+2|B|+\left|T(\lambda)_{v}^{x}\right|}
\end{array}
$$

where $B$ runs over all the latticed subsets for $T(\lambda)_{v}^{x}$. Using arguments similar to the above, we get $a_{\lambda \uparrow^{v}}(q)=[1+|T|]$, and $\sum_{B} q^{\left.1+2|B|+\mid T(\lambda)_{v}^{x}\right) \mid}=d_{\lambda \uparrow^{v}, \lambda \uparrow^{x}}(q)$ for all $x \in N^{-}(T)$.

Now, let $\rho=\lambda \uparrow^{i_{1}} \downarrow_{j_{1}} \ldots \uparrow^{i_{s}} \downarrow_{j_{s}}$, with $i_{1}<j_{1}<\cdots<i_{s}<j_{s}<v$. Then by the row removal theorem (Theorem 2.5(1)), we have

$$
\begin{aligned}
\left.\left\langle f_{r}(G(\lambda)), s\left(\rho \uparrow^{v}\right)\right)\right\rangle & =\sum_{x \in S^{+}(T)} d_{\rho \uparrow^{v} \downarrow_{x}, \lambda}(q)\left\langle f_{r}\left(s\left(\rho \uparrow^{v} \downarrow_{x}\right)\right), s\left(\rho \uparrow^{v}\right)\right\rangle \\
& =\sum_{x \in S^{+}(T)} d_{\rho \lambda}(q) d_{\lambda \uparrow^{v} \downarrow_{x}, \lambda}(q)\left\langle f_{r}\left(s\left(\lambda \uparrow^{v} \downarrow_{x}\right)\right), s\left(\lambda \uparrow^{v}\right)\right\rangle \\
& =d_{\rho \lambda}(q)\left\langle f_{r}(G(\lambda)), s\left(\lambda \uparrow^{v}\right)\right\rangle \\
& =d_{\rho \lambda}(q) \sum_{y \in N^{-}(T) \cup\{v\}} a_{\lambda \uparrow^{y}}(q) d_{\lambda \uparrow^{v}, \lambda \uparrow^{y}}(q) \\
& =\sum_{y \in N^{-}(T) \cup\{v\}} a_{\lambda \uparrow^{y}}(q) d_{\rho \uparrow^{v}, \lambda \uparrow^{y}}(q) \\
& =\left\langle\sum_{y \in N^{-}(T) \cup\{v\}} a_{\lambda \uparrow^{y}}(q) G\left(\lambda \uparrow^{y}\right), s\left(\rho \uparrow^{v}\right)\right\rangle .
\end{aligned}
$$


This implies that $\left\langle a_{\rho \uparrow^{v}}(q) G\left(\rho \uparrow^{v}\right), s\left(\rho \uparrow^{v}\right)\right\rangle=0$ by Proposition 2.4 so that $a_{\rho \uparrow^{v}}(q)=$ 0 . Thus Theorem 4.1 holds for $v$.

Now, suppose $\mu \in \mathcal{P}_{n+1}$. Note that the decomposition number $d_{\mu \uparrow^{v} \downarrow_{w}, \mu}(q)$ can be obtained by the induction hypothesis and Theorem 2.5, except when $w$ indexes the removable node in the top row of the Young diagram of $\mu$ and $v$ indexes the bottom indent node in the first column. For this case, let $\lambda=\mu \downarrow_{w}$; then we have seen above that

$$
\begin{aligned}
& d_{\mu \uparrow^{v} \downarrow_{w}, \mu}(q)=d_{\lambda \uparrow^{v}, \lambda \uparrow^{w}}(q) \\
& =\sum_{B} q^{1+2|B|+\left|T(\lambda)_{v}^{w}\right|} \\
& =\sum_{A} q^{1+2|A|+\left|T(\mu)_{v}^{w}\right|},
\end{aligned}
$$

where $A$ runs over the latticed subsets for $T(\mu)_{v}^{w}$. Thus Theorem 4.2 holds for $\mu$, and this completes our proof.

We thank the referee for the following remark.

Remark. Since both $d_{\lambda \mu}(q)$ and the decomposition numbers of symmetric groups obey the row removal theorem, it follows from Theorem 4.2. Theorem 1.10 of [6] and Ariki's theorem 1 that the decomposition number indexed by partitions $\lambda$ and $\mu$ (with $\mu$-regular) of the Hecke algebra at a complex $p$-th root of unity coincide with that of the symmetric group in characteristic $p$ when $\lambda$ is obtained from $\mu$ by alternately removing removable nodes and adding indent nodes. This further implies that the corresponding adjustment matrix entry indexed by such a pair of partitions is zero. As Theorem 1.10 of 6 can be extended to an analogue for the Schur algebras, the adjustment matrix entry, from the quantized Schur algebra at a complex $p$-th root of unity to the classical Schur algebra in characteristic $p$, indexed by such a pair of partitions (where $\mu$ need not be $p$-regular), is also zero.

We conclude this paper with an example illustrating the main theorems.

Example. Let $e=2, \lambda=\left(5,3^{2}, 1\right)$, and $r=0$.

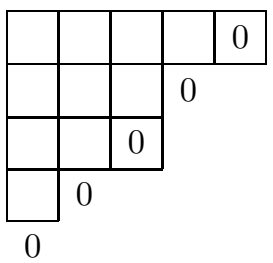

Young diagram of $\lambda=\left(5,3^{2}, 1\right)$.

Then $T(\lambda)=(-1,-1,1,-1,1)$. Let $f_{0}(G(\lambda))=\sum_{\mu} a_{\mu}(q) G(\mu)$. Then

$$
\begin{gathered}
a_{(5,4,3,1)}(q)=a_{\left(5,3^{2}, 2\right)}(q)=[2]=q+q^{-1}, \\
a_{\left(5,3^{2}, 1^{2}\right)}(q)=a_{\left(5,4,2^{2}\right)}(q)=a_{\left(5,4,2,1^{2}\right)}(q)=0,
\end{gathered}
$$


and

$$
\begin{gathered}
d_{\left(4^{2}, 3,1\right),\left(5,3^{2}, 1\right)}(q)=d_{\left(5,3,2^{2}\right),\left(5,3^{2}, 1\right)}(q)=q, \\
d_{\left(5,3,2,1^{2}\right),\left(5,3^{2}, 1\right)}(q)=q^{2}, \\
d_{\left(4,3^{2}, 2\right),\left(5,3^{2}, 1\right)}(q)=q+q^{3}, \\
d_{\left(4,3^{2}, 1^{2}\right),\left(5,3^{2}, 1\right)}(q)=q^{2}+q^{4} .
\end{gathered}
$$

Using the row removal theorem (Theorem 2.5(1)), we further have

$$
d_{\left(4^{2}, 2^{2}\right),\left(5,3^{2}, 1\right)}(q)=q^{2} \quad \text { and } \quad d_{\left(4^{2}, 2,1^{2}\right),\left(5,3^{2}, 1\right)}(q)=q^{3} .
$$

\section{ACKNOWLEDGEMENT}

We thank Bernard Leclerc for his comment on generalising the results of this paper. The second author thanks Yoshihisa Saito for the helpful discussions on the relationship between canonical bases and perverse sheaves. The third author acknowledges support by the Academic Research Fund R-146-000-043-112 of the National University of Singapore.

\section{REFERENCES}

[1] S. Ariki. On the decomposition numbers of the Hecke algebra of $G(m, 1, n)$. J. Math. Kyoto Univ., 36(4):789-808, 1996. MR1443748 (98h:20012)

[2] J. Chuang, H. Miyachi, and K.M. Tan. Row and column removal in the $q$-deformed Fock space. J. Algebra, 254:84-91, 2002. MR1927432 (2003h:17018)

[3] T. Hayashi. $q$-analogues of Clifford and Weyl algebras - spinor and oscillator representations of quantum enveloping algebras. Comm. Math. Phys., 127(1):129-144, 1990. MR:1036118 (91a:17015)

[4] G. D. James and A. Kerber. The representation theory of the symmetric group, volume 16 of Encyclopedia of Mathematics and its Applications. Addison-Wesley Publishing Co., Reading, Mass., 1981. MR0644144 (83k:20003)

[5] M. Kashiwara. On crystal bases of the $Q$-analogue of universal enveloping algebras. Duke Math. J., 63(2):465-516, 1991. MR1115118 (93b:17045)

[6] A. Kleshchev. On decomposition numbers and branching coefficients for symmetric and special linear groups. Proc. London Math. Soc. (3), 75:497-558, 1997. MR1466660 (98g:20026)

[7] A. Lascoux, B. Leclerc, and J.-Y. Thibon. Hecke algebras at roots of unity and crystal bases of quantum affine algebras. Comm. Math. Phys., 181(1):205-263, 1996. MR1410572 (97k:17019)

[8] B. Leclerc. Symmetric functions and the Fock space representation. Symmetric functions 2001: Surveys of developments and perspectives, 153-177, NATO Sci. Ser. II Math. Phys. Chem., 74, Kluwer Acad. Publ., Dordrecht, 2002. MR2059362 (2005g:05151)

[9] B. Leclerc and J.-Y. Thibon. Canonical bases of $q$-deformed Fock spaces. Internat. Math. Res. Notices, (9):447-456, 1996. MR.1399410(97h:17023)

[10] G. Lusztig. Canonical bases arising from quantized enveloping algebras. J. Amer. Math. Soc. (3):447-498, 1990. MR 1035415 (90m:17023)

[11] G. Lusztig. Quivers, perverse sheaves and quantized enveloping algebras. J. Amer. Math. Soc. (4):365-421, 1991. MR:1088333 (91m:17018)

[12] G. Lusztig. Canonical bases and Hall algebras. In Representation Theories and Algebraic Geometry, A. Broer and A. Daigneault, eds. NATO ASI series (514) pp.365-399, 1998. MR:1653038 (2000d:17020)

[13] A. Mathas. Iwahori-Hecke algebras and Schur algebras of the symmetric group, University Lecture Series, vol. 15, American Mathematical Society, Providence, RI, 1999. MR:1711316 (2001g:20006)

[14] K. Misra and T. Miwa. Crystal base for the basic representation of $U_{q}(\mathfrak{s l}(n))$. Comm. Math. Phys., 134(1):79-88, 1990. MR1079801 (91j:17021) 
[15] O. Schiffmann. The Hall algebra of a cyclic quiver and canonical bases of Fock spaces, Internat. Math. Res. Notices, 8:413-440, 2000. MR.1753691 (2001g:16032)

[16] M. Varagnolo and E. Vasserot. On the decomposition matrices of the quantized Schur algebra. Duke Math. J., 100(2):267-297, 1999. MR1722955 (2001c:17029)

Department of Mathematics, University of Bristol, University Walk, Bristol BS8 1TW, UniTED KINGDOM

E-mail address: joseph.chuang@bris.ac.uk

Graduate School of Mathematics, Nagoya University, Chikusa-ku, Nagoya 464-8602, JAPAN

E-mail address: miyachi@math.nagoya-u.ac.jp

Department of Mathematics, National University of Singapore, 2, Science Drive 2, SiNGAPORE 117543

E-mail address: tankm@nus.edu.sg 